\section{High-Quality Genome Assembly of Pseudopestalotiopsis theae, the Pathogenic Fungus Causing Tea Gray Blight}

\author{
Shiqin Zheng, ${ }^{1}$ Ruiqi Chen, ${ }^{1}$ Zhe Wang, ${ }^{2}$ Juan Liu, ${ }^{2}$ Yan Cai, ${ }^{1}$ Minghui Peng, ${ }^{1}$ Tian Zhang, ${ }^{1}$ \\ Yunxi $\mathrm{Li}^{1}{ }^{1}$ Baohua Wang, ${ }^{1}$ Jiandong Bao, ${ }^{2}$ Dongmei Zhang, ${ }^{1}$ Zonghua Wang, ${ }^{1,3,+}$ and Hongli $\mathrm{Hu}^{1, \dagger}$ \\ ${ }^{1}$ Fujian Universities Key Laboratory for Plant-Microbe Interaction, College of Plant Protection, Fujian \\ Agriculture and Forestry University, Fuzhou, 350002, China \\ ${ }^{2}$ Fujian Universities Key Laboratory for Plant-Microbe Interaction, College of Life Sciences, Fujian \\ Agriculture and Forestry University, Fuzhou, 350002, China \\ ${ }^{3}$ Institute of Oceanography, Minjiang University, Fuzhou, 350108, China
}

\begin{abstract}
Tea gray blight is one of the most serious foliar diseases of tea tree, caused by the plantpathogenic fungus Pseudopestalotiopsis theae, which can affect production and quality of tea worldwide. We generated a highly contiguous, 50.41-Mbp genome assembly $\left(\mathrm{N}_{50}=1.30 \mathrm{Mbp}\right)$ of $P$. theae strain CYF27 by combining PacBio long-read and Illumina short-read sequencing technologies. We identified a total of 15,626 gene models, of which 1,038 genes encode putative secreted proteins. The high-quality genome assembly and annotation resource reported here will be useful for the study of fungal infection mechanisms and pathogen-host interaction.
\end{abstract}

\section{Genome Announcement}

Tea gray blight is one of the most serious foliar diseases of tea plants (Camellia sinensis $L$.) (Wang et al. 2019), which causes damage to the production and quality of tea. This disease is caused by Pseudopestalotiopsis theae (Sawada) Maharachch., K. D. Hyde and Crous, which mainly infects mature and old foliage and can also infect young shoots (Maharachchikumbura et al. 2014). P. theae causes defoliation of tea trees under high temperature and high humidity, leading to poor growth and tea yield reduction (Wang and Lu 2008). Tea gray blight was reported for the first time in Kagoshima, Japan in 1973 and called zonate leaf spot (Takaya 1978). There are two dominant pathogens: Pestalotiopsis theae (Sawada) Steyaert and Pestalotiopsis longiseta (Speg.) K. Dai \& Tak. Kobay. P. theae was renamed as Pseudopestalotiopsis theae in 2014 (Maharachchikumbura et al. 2014), and is widely found in tea-growing countries such as India and China, while Pestalotiopsis longiseta is widely found in Japan (Joshi et al. 2009; Nagata et al. 1992; Takeda 2002).

Recent reports confirmed that Pseudopestalotiopsis spp. are not only common phytopathogens that cause a variety of diseases but also endophytes that produce kinds of bioactive natural products (Maharachchikumbura et al. 2011). Until now, only two Pseudopestalotiopsis spp. have been whole-genome sequenced (i.e., Pseudopestalotiopsis fici and Pseudopestalotiopsis sp. JCM 9685, endophytes of $C$. sinensis and Taxus brevifolia, respectively) (Pulici et al. 1997; Wang et al. 2015). However, the genome assembly of a pathogenic Pseudopestalotiopsis sp. has not yet been released. The genome assembly of pathogenic fungus $P$. theae strain CYF27 generated here will support the analysis of pathogenic mechanisms and interaction mechanisms with the host systematically at the molecular level.

${ }^{\dagger}$ Corresponding authors: Z. Wang; wangzh@fafu.edu.cn; and H. Hu; huhongli7905@gmail.com

S. Zheng and R. Chen contributed equally to this work.

The author(s) declare no conflict of interest.

Accepted for publication 9 May 2021.

\section{Funding}

This research is supported by Natural Science Foundations of China (31800008 and U1803232), Natural Science Foundation of Fujian Province, China (2019J01385), Outstanding Young Scientific Research Project of Fujian Agriculture and Forestry University (XJQ201511), and Fujian Agriculture and Forestry University Construction Project for Technological Innovation and Service System of Tea Industry Chain (K1520005A03).

\section{Keywords}

fungal pathogen, genome, Pseudopestalotiopsis theae, tea grey blight 
Table 1. Genome characteristics of strain CYF27

\begin{tabular}{|c|c|}
\hline Features & Values \\
\hline \multicolumn{2}{|l|}{ Reads features (PacBio Sequel) } \\
\hline Total reads (Gbp) & 16.65 \\
\hline Average read length (Kbp) & 8.67 \\
\hline Reads $\mathrm{N}_{50}(\mathrm{Kbp})$ & 10.24 \\
\hline Maximum read length (Kbp) & 65.75 \\
\hline \multicolumn{2}{|l|}{ Genome features } \\
\hline Heterozygosity (\%) ${ }^{a}$ & 0.01 \\
\hline Predicted genome size $(\mathrm{Mbp})^{\mathrm{a}}$ & 51.65 \\
\hline Assembly size (Mbp) & 50.41 \\
\hline Coverage $(x)$ & 323 \\
\hline GC content $(\%)$ & 49.5 \\
\hline Contig number & 61 \\
\hline Contig $\mathrm{N}_{50}(\mathrm{Mbp})$ & 1.30 \\
\hline Average contig length (Mbp) & 0.81 \\
\hline Maximum contig length (Mbp) & 3.56 \\
\hline Repeat sequence $(\%)$ & $1.97(\text { TE: } 0.49)^{\mathrm{c}}$ \\
\hline Protein-coding genes & 15,626 \\
\hline Candidate secreted proteins & 1,038 \\
\hline Genes annotated by InterProScan and eggNOG-mapper ${ }^{\mathrm{b}}$ & 12,230 \\
\hline SMGCs $^{d}$ & 64 \\
\hline \multicolumn{2}{|c|}{$\begin{array}{l}\text { a Genome size and heterozygosity was estimated by GenomeScope combined with Jellyfish using } \\
\text { Illumina short reads. } \\
\text { b Protein Families Database (Pfam) annotated by InterProScan, and Gene Ontology (GO), Kyoto } \\
\text { Encyclopedia of Genes and Genomes (KEGG), carbohydrate active enzyme (CAZy), and Clusters } \\
\text { of Orthologous Groups (COG) annotated by eggNOG-mapper. } \\
\text { c TE = transposable elements. } \\
\text { d Secondary metabolite gene clusters (SMGCs) annotated by AntiSMASH. }\end{array}$} \\
\hline
\end{tabular}

In this study, $P$. theae strain CYF27 was isolated from an infected leaf of tea plants in the main campus of Fujian Agriculture and Forestry University, Fujian Province, China. This strain was identified to $P$. theae by combining morphological data and multiloci phylogenetic analyses (internal transcribed spacer, $\beta$-tubulin, and translation elongation factor) (Chen et al. 2018; Khan et al. 2011; Liu et al. 2017; Maharachchikumbura et al. 2013).

Genome sequencing was performed by both the first-generation PacBio Sequel system and Illumina Hiseq 2500 platform at Beijing Novogene Bioinformatics Technology Co. Ltd. (Beijing, China). For PacBio long-read sequencing, a $20-\mathrm{Kb}$ genomic library was prepared and generated using one single-molecule real-time (SMRT) cell of data. After filtering the low-quality and short reads ( $<1 \mathrm{Kbp}$ ), we finally obtained $16.65 \mathrm{Gbp}$ of PacBio raw reads (323x coverage), with a maximum length of $65.75 \mathrm{Kbp}$, an average length of $8.67 \mathrm{Kbp}$, and an $\mathrm{N}_{50}$ length of $10.24 \mathrm{Kbp}$ (Table 1). For Illumina short-read sequencing, a 300-bp genomic library was PE150 (150 bp paired-end) sequenced and generated 3.97 Gbp short reads (45x coverage).

Genome features, including genome size, heterozygosity, and repeat content, are key parameters for genome assembly. K-mer-based genome size estimation was performed by Kmergenie (V1.7048, http://kmergenie.bx.psu.edu/, diploid model, best $k=55$ ) with Illumina short reads, which showed that the expected genome length was $51.65 \mathrm{Mbp}$ (Table 1). Subsequently, genome heterozygosity $(0.01 \%)$ and repeat content $(2.11 \%)$ were estimated by GenomeScope (http://qb.cshl.edu/genomescope/) combined with Jellyfish (V2.2.4) using Illumina short reads (Table 1).

A 50.41-Mbp draft genome assembly of $P$. theae strain CYF27 was de novo assembled by HGAP4 pipeline in SMRT Link V6 (expected genome size $=51 \mathrm{Mbp}$ ) using pure PacBio long reads (Bitar et al. 2019). The assembly size was consistent with the predicted genome size generated by Kmergenie. Then, two rounds of genome polishing were conducted by NextPolish (V1.2.4, https://github.com/Nextomics/NextPolish, task = best) with both PacBio long reads and Illumina short reads. The polished genome assembly consisted of 61 contigs with an average contig length of $0.81 \mathrm{Mbp}$, a maximum contig length of $3.56 \mathrm{Mbp}$, and a contig $\mathrm{N}_{50}$ length of 1.30 Mbp (Table 1).

RepeatModeler v1.08 and RepeatMasker v4.0.9 (http://www.repeatmasker.org) were applied together to do repeat identification and found that $1.97 \%$ of the genome was inferred 

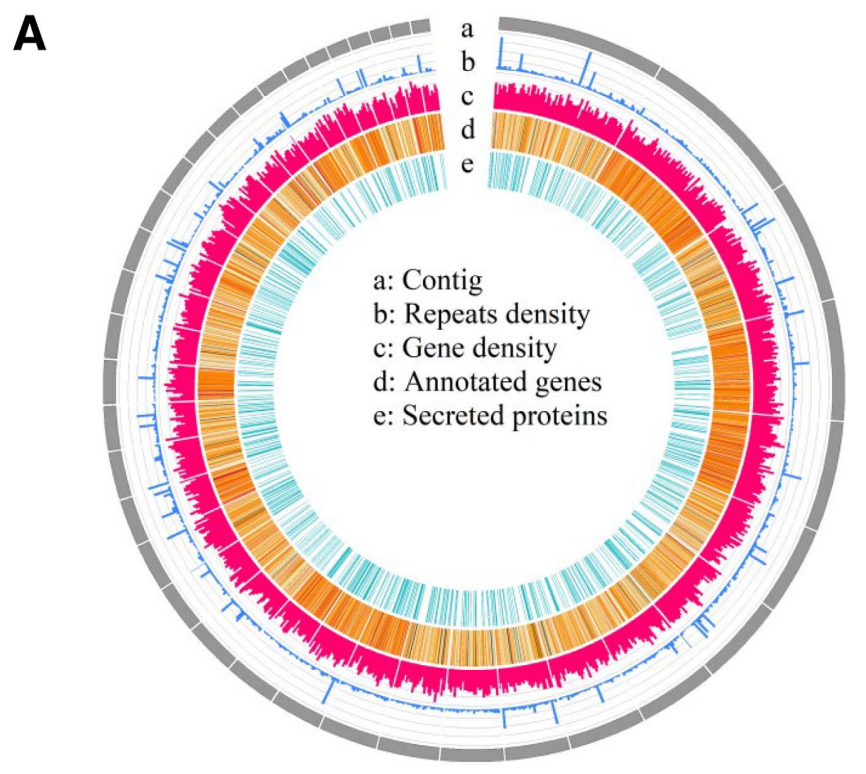

B

Betalactone
Fungal-RiPP
Indole
NRPS
NRPS-like
T1PKS
T3PKS
$\square$ Terpene

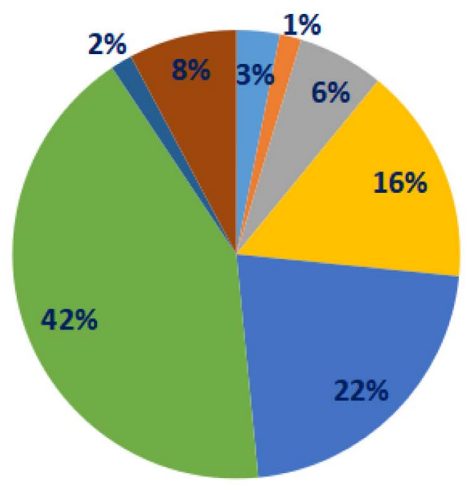

\begin{tabular}{|c|c|}
\hline 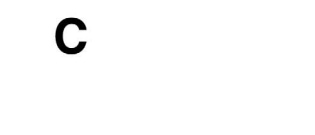 & $\begin{array}{l}\text { Complete (C) and single-copy (S) } \\
\text { Complete (C) and duplicated (D) } \\
\text { Fragment }(\mathrm{F}) \\
\text { Missing }(\mathrm{M})\end{array}$ \\
\hline Genome_ascomycota & C: 1672 [S:1666, D:6], F:6, M:28, n:1706 \\
\hline Genome_fungi & C: 746 [S:745, D:1], F:0, M:12, n:758 \\
\hline Genome sordariomycetes & C: 3784 [S:3769, D:15], F:3, M:30, n:3817 \\
\hline Protein_ascomycota & C: 1689 [S:1668, D:21] F:5, M:12, n:1706 \\
\hline Protein_fungi & C: 751 [S:745, D:6], F:2, M:5, n:758 \\
\hline \multirow[t]{2}{*}{ Protein_sordariomycetes } & C: 3760 [S:3684, D:76], F:13, M:44, n:3817 \\
\hline & 60 \\
\hline & $\% \mathrm{BUSCOs}$ \\
\hline
\end{tabular}

Fig. 1. Schematic diagrams of the sequencing, assembly, and annotation of Pseudopestalotiopsis theae strain CYF27 genome. A, Genome features of $P$. theae strain CYF27 shown by Circos (http://circos.ca/). a: Contigs (>100 Kbp). b: Repeats calculated by sliding window analysis (50 Kbp). c: Density of genes. d: Genes with functional annotation. e: Putative secreted proteins. B, Secondary metabolite gene clusters. FungalRiPP = Fungal RiPP with POP or UstH peptidase types and a modification, NRPS = nonribosomal peptide synthetase cluster, and T1PKS = type I polyketide synthase. C, Completeness of genome assembly was assessed by BUSCO v4.1.4 with single-copy orthologs from OrthoDB v10 at Fungi $(n=758)$, Ascomycota $(n=1706)$, and Sordariomycetes $(n=3817)$ levels.

to consist of repeats, of which only $0.49 \%$ was transposable elements (Table 1 ). The repeat masked genome assembly was used for protein-coding gene prediction conducted by BRAKER2 (Hoff et al. 2019). Combing ab initio gene predictions, evidence from RNA sequencing data, and homologous proteins, we found a total of 15,626 protein-coding genes. Of those genes, 1,038 candidate secreted proteins were identified by SignalP V5.0 and TMHMM V2.0 following our previous pipeline (Bao et al. 2017). Gene functional annotation was performed by InterProScan v5.46-81.0 (https://github.com/ebi-pf-team/interproscan/) and eggNOG-mapper v2 (http://eggnog-mapper.embl.de/) (Fig. 1A), and found that, in total, 12,230 genes (78.3\%) could be annotated by Kyoto Encyclopedia of Genes and Genomes (https://www.kegg.jp/), Protein Families Database (http://pfam.xfam.org/), Gene Ontology (http://geneontology.org/), InterPro (https://www.ebi.ac.uk/interpro/), and carbohydrate active enzymes (www.cazy.org) (Table 1). AntiSMASH v5.2.0 (https://fungismash.secondarymetabolites.org/) identified 64 secondary metabolite gene clusters, including $2 \beta$-lactones, 1 fungal-RiPP, 1 indole, 10 nonribosomal peptide synthetase clusters (NRPSs), 14 NRPS-likes, 27 type I polyketide synthases (PKSs), 1 type III PKS, and 5 terpenes (Fig. 1B).

The completeness of the genome assembly and predicted genes were assessed by BUSCO v4.1.2 (Seppey et al. 2019) with Fungi $(n=758)$, Ascomycota $(n=1,706)$, and 
Sordariomycetes lineage $(n=3,817)$ datasets from OrthoDB v10 (https://www.orthodb.org/). The genome assembly contains 746 (98.4\%), 1,672 (98.1\%), and 3,784 (99.1\%) complete orthologs in Fungi, Ascomycota, and Sordariomycetes, respectively (Fig. 1C), while gene predictions showed 99.1, 99.0, and 98.5\% completeness with 751, 1,689, and 3,760 complete orthologs in Fungi, Ascomycota, and Sordariomycetes, respectively.

The genome assembly and gene annotations data have been deposited at GenBank under the accession number JACWGD000000000.

\section{Acknowledgments}

We thank J. Norvienyeku for assistance with manuscript preparation.

\section{Literature Cited}

Bao, J. D., Chen, M. L., Zhong, Z. H., Tang, W., Lin, L. Y., Zhang, X. T., Jiang, H. L., Zhang, D. M., Miao, C. Y., Tang, H. B., Zhang, J. S., Lu, G. D., Ming, R., Norvienyeku, J., Wang, B. H., and Wang, Z. H. 2017. PacBio sequencing reveals transposable elements as a key contributor to genomic plasticity and virulence variation in Magnaporthe oryzae. Mol. Plant 10:1465-1468.

Bitar, I., Moussa, J., Abboud, E., Hrabak, J., and Tokajian, S. 2019. Integration of two pKPX-2-derived antibiotic resistance islands in the genome of an ESBLproducing Klebsiella pneumoniae ST3483 from Lebanon. J. Glob. Antimicrob. Resist. 18:257-259.

Chen, Y. J., Zeng, L., Shu, N., Jiang, M. Y., Wang, H., Huang, Y. J., and Tong, H. R. 2018. Pestalotiopsis-like species causing gray blight disease on Camellia sinensis in China. Plant Dis. 102:98-106.

Hoff, K. J., Lomsadze, A., Borodovsky, M., and Stanke, M. 2019. Whole-genome annotation with BRAKER. Pages 5-95 in: Gene Prediction. M. Kollmar, ed. Methods in Molecular Biology, vol. 1962. Humana, New York, NY, U.S.A.

Joshi, D. S., Sanjay, R., Baby, U. I., and Mandal, A. K. A. 2009. Molecular characterization of Pestalotiopsis spp. associated with tea (Camellia sinensis) in southern India using RAPD and ISSR markers. Indian J. Biotechnol. 8:377-383.

Khan, A. L., Hamayun, M., Kim, Y. H., Kang, S. M., Lee, J. H., and Lee, I. J. 2011. Gibberellins producing endophytic Aspergillus fumigatus sp. LHO2 influenced endogenous phytohormonal levels, isoflavonoids production and plant growth in salinity stress. Process Biochem. 46:440-447.

Liu, F., Hou, L. W., Raza, M., and Cai, L. 2017. Pestalotiopsis and allied genera from Camellia, with description of 11 new species from China. Sci. Rep. 7:866.

Maharachchikumbura, S. S. N., Chukeatirote, E., Guo, L.-D., Crous, P. W., Mckenzie, E. H. C., and Hyde, K. D. 2013. Pestalotiopsis species associated with Camellia sinensis (tea). Mycotaxon 123:47-61.
Maharachchikumbura, S. S. N., Guo, L. D., Chukeatirote, E., Bahkali, A. H., and Hyde, K. D. 2011. Pestalotiopsis-morphology, phylogeny, biochemistry and diversity. Fungal Divers. 50:167-187.

Maharachchikumbura, S. S. N., Hyde, K. D., Groenewald, J. Z., Xu, J., and Crous, P. W. 2014. Pestalotiopsis revisited. Stud. Mycol. 79:121-186.

Nagata, T., Ando, Y., and Hirota, A. 1992. Phytotoxins from tea gray blight fungi, Pestalotiopsis longiseta and Pestalotiopsis theae. Biosci. Biotechnol. Biochem. 56:810-811.

Pulici, M., Sugawara, F., Koshino, H., Okada, G., Esumi, Y., Uzawa, J., and Yoshida, S. 1997. Metabolites of Pestalotiopsis spp., endophytic fungi of Taxus brevifolia. Phytochemistry 46:313-319.

Seppey, M., Manni, M., and Zdobnov, E. M. 2019. BUSCO: Assessing genome assembly and annotation completeness. Pages 227-245 in: Gene Prediction. M. Kollmar, ed. Methods in Molecular Biology, vol. 1962. Humana, New York, NY, U.S.A. , and

Takaya, S. 1978. New diseases of tea plant. JARQ 12:138-140.

Takeda, Y. 2002. Genetic analysis of tea gray blight resistance in tea plants. Jpn. Agric. Res. Q. 36:143-150.

Wang, J. P., and Lu, D. S. 2008. Occurrence of tea grey spot disease and germinating characteristics of Pestalotiopsis versicolor conidiospores. Anjisuan $\mathrm{He}$ Shengwu Ziyuan (Amino Acids and Biological Resources) 30:30-32 (in Chinese).

Wang, S. S., Mi, X. Z., Wu, Z. R., Zhang, L. X., and Wei, C. L. 2019. Characterization and pathogenicity of Pestalotiopsis-like species associated with gray blight disease on Camellia sinensis in Anhui Province, China. Plant Dis. 103:2786-2797.

Wang, X. N., Zhang, X. L., Liu, L., Xiang, M. C., Wang, W. Z., Sun, X., Che, Y. S., Guo, L. D., Liu, G., Guo, L. Y., Wang, C. S., Yin, W. B., Stadler, M., Zhang, X. Y., and Liu, X. Z. 2015. Genomic and transcriptomic analysis of the endophytic fungus Pestalotiopsis fici reveals its lifestyle and high potential for synthesis of natural products. BMC Genomics 16:28-40. 